\title{
Analytical expressions of the concentrations of substrate and product in enzyme inhibition process
}

\author{
Sankaranarayanan Muthukumar, Lakshmanan Rajendran \\ Department of Mathematics, The Madura College, Madurai, India; raj_sms@rediffmail.com
}

Received 3 June 2013; revised 3 July 2013; accepted 10 July 2013

Copyright (C 2013 Sankaranarayanan Muthukumar, Lakshmanan Rajendran. This is an open access article distributed under the Creative Commons Attribution License, which permits unrestricted use, distribution, and reproduction in any medium, provided the original work is properly cited.

\begin{abstract}
The initial and boundary value problem in enzyme reactions mechanism for inhabitation process is discussed. Approximate analytical expressions for the concentrations of substrate and product are presented. Approximate analytical solutions of non-linear reaction equations containing non-linear terms related to enzymatic reaction mechanism are solved using Homotopy perturbation method. The relevant analytical expression for the substrate and product concentration profiles is discussed in terms of dimensionless reaction diffusion parameters $\alpha, \beta, \gamma_{E}$, and $\gamma_{s}$. Numerical solution is also obtained using Matlab program. Our analytical expression compared with numerical estimation and good agreement is noted.
\end{abstract}

Keywords: Initial and Boundary Value Problems; Enzyme Kinetics; Non-Linear Reaction Equations; Homotopy Perturbation Method; Michaelis-Menten Kinetics

\section{INTRODUCTION}

Enzymes are catalysts and increase the speed of a chemical reaction without themselves undergoing any permanent chemical change [1-7]. They are neither used up in the reaction nor do they appear as reaction products. An enzyme enhances the rate of the reaction it influences. The catalysts is not consumed as a result of the reaction, nor does it alter the equilibrium constant [8,9]. In this two-step reaction, the enzyme combines with substrate to form the complex, which can either dissociate again into unchanged substrate and enzyme or go on to the second step and form the products and unchanged enzyme. The reverse of the second step would lead to the synthesis of ES from enzyme and products, but this process can gen- erally be ignored unless the products are allowed to accumulate. Also that the total substrate and product concentration in the enzyme kinetics in biochemical systems has been modeled by system of nonlinear ordinary differential equations.

An enzyme inhibitor is a molecule, which binds to enzymes and decreases their activity. Since blocking an enzyme's activity can kill a pathogen or correct a metabolic imbalance, many drugs are enzyme inhibitors. They are also used as herbicides and pesticides. Not all molecules that bind to enzymes are inhibitors; enzyme activators bind to enzymes and increase their enzymatic activity, while enzyme substrates bind and are converted to products in the normal catalytic cycle of the enzyme.

The binding of an inhibitor can stop a substrate from entering the enzyme's active site. Inhibitor binding is either reversible or irreversible. Irreversible inhibitors usually react with the enzyme and change it chemically. In contrast, reversible inhibitors bind non-covalently and different types of inhibition are produced depending on whether these inhibitors bind to the enzyme, the enzymesubstrate complex, or both. Many drug molecules are enzyme inhibitors, so their discovery and improvement is an active area of research in biochemistry and pharmacology.

Mathematical modeling of enzyme kinetics is given in the books by Rubinow [10], Murray [11], Segel [12] and Roberts [13]. Recently Rajendran and his team also solved some non linear problems in enzyme reaction kinetics [14-19]. The purpose of this communication is to derive asymptotic approximate expressions for the concentration of substrate and product in enzyme inhibition process.

\section{MATHEMATICAL FORMULATION AND ANALYSIS OF THE SUBSTRATE INHIBITION MODELS}

The substrate act as an inhibitor for enhancing the rate 
of reaction. The scheme of the enzyme $(E)$ catalysed conversion of the substrate $(S)$ to the product $(P)$

$$
S \rightarrow P
$$

is a simplified version of

$$
E+S \leftrightarrow E S \rightarrow E+P
$$

According to scheme (2), the substrate $(S)$ combines reversibly with the enzyme $(E)$ to form the enzyme-substrate complex $(E S)$. The complex then dissociates into product $(P)$ and the enzyme is regenerated. The simplest scheme of non-Michaelis-Menten kinetics may be, for example, described by adding the relationship of the interaction of the enzyme substrate complex $(E S)$ with another substrate molecule $(S)$ followed by the generation of the non-active complex (ESS) to the Michaelis-Menten scheme

$$
E S+S \leftrightarrow E S S
$$

The above substrate inhibition process can be represented by the following system of nonlinear mass balance equations:

$$
\begin{gathered}
\frac{\partial S}{\partial t}=D_{S} \frac{\partial^{2} S}{\partial x^{2}}-\frac{V_{\max } S}{K_{M}+S\left(1+S / K_{S}\right)} \\
\frac{\partial P}{\partial t}=D_{P} \frac{\partial^{2} P}{\partial x^{2}}+\frac{V_{\max } S}{K_{M}+S\left(1+S / K_{S}\right)}, S \in(0, d), t>0
\end{gathered}
$$

where $x$ and $t$ stand for space and time, respectively, $S(x, t)$ is the concentration of the substrate, $P(x, t)$ is the concentration of the reaction product, $d$ is the thickness of the enzyme layer, $D_{S}, D_{P}$ are the diffusion coefficients, $V_{\max }$ is the maximal enzyme rate and $K_{M}$ and $K_{S}$ are the Michaelis and the substrate inhibition constants, respectively. The governing Eq.4 together with the following initial and the boundary conditions form the initial boundary value problem $(t>0)$

$$
S(x, 0)=0 \text { and } P(x, 0)=0
$$

The boundary conditions are:

$$
\begin{aligned}
& S(d, 0)=S_{0}, S(d, t)=S_{0}, \\
& P(0, t)=p(d, t)=0,\left.\quad \frac{\partial S}{\partial x}\right|_{x=0}=0,
\end{aligned}
$$

At steady state the Eqs. $\mathbf{4}$ and $\mathbf{5}$ becomes

$$
\begin{aligned}
& D_{S} \frac{\mathrm{d}^{2} S}{\mathrm{~d} x^{2}}-\frac{V_{\text {max }} S}{K_{M}+S\left(1+S / K_{S}\right)}=0 \\
& D_{P} \frac{\mathrm{d}^{2} P}{\mathrm{~d} x^{2}}+\frac{V_{\text {max }} S}{K_{M}+S\left(1+S / K_{S}\right)}=0
\end{aligned}
$$

with the boundary conditions

$$
\begin{gathered}
P(x=0)=0, \frac{\mathrm{d} S}{\mathrm{~d} x}=0 \\
P(x=d)=0, S(x=d)=S_{0}
\end{gathered}
$$

The density of the biosensor current is given by:

$$
i=\left.n_{e} F D_{P} \frac{\partial P}{\partial x}\right|_{x=0}
$$

The following sets of dimensionless variables are introduced:

$$
\begin{aligned}
& X=\frac{x}{d}, \bar{S}=\frac{S}{S_{0}}, \bar{P}=\frac{P}{S_{0}}, \gamma_{E}^{2}=\frac{\mathrm{d}^{2} V_{\max }}{D_{S} K_{M}}, \\
& \alpha=\frac{S_{0}}{K_{M}}, \beta=\frac{S_{0}^{2}}{K_{M} K_{S}}, \gamma_{S}^{2}=\frac{\mathrm{d}^{2} V_{\max }}{D_{P} K_{M}}
\end{aligned}
$$

Thus dimensionless Eqs. 8 and 9 becomes

$$
\begin{aligned}
& \frac{\mathrm{d}^{2} \bar{S}}{\mathrm{~d} X^{2}}-\frac{\gamma_{E}^{2} \bar{S}}{\left(1+\alpha \bar{S}+\beta \bar{S}^{2}\right)}=0 \\
& \frac{\mathrm{d}^{2} \bar{P}}{\mathrm{~d} X^{2}}+\frac{\gamma_{S}^{2}}{\left(1+\alpha \bar{S}+\beta \bar{S}^{2}\right)}=0
\end{aligned}
$$

The dimensionless boundary conditions becomes

$$
\begin{aligned}
& X=0, \bar{P}=0, \frac{\mathrm{d} \bar{S}}{\mathrm{~d} x}=0 \\
& X=1, \bar{P}=0, \bar{S}=1
\end{aligned}
$$

The dimensionless current is given by

$$
\psi=\frac{i d}{n_{e} F D_{P} s_{0}}=\left.\frac{\mathrm{d} \bar{P}}{\mathrm{~d} X}\right|_{X=0}
$$

\section{ANALYTICAL EXPRESSION OF CONCENTRATIONS OF SUBSTRATE AND PRODUCT USING HPM}

After recently, many authors have applied the HPM to solve the non-linear problem in physics and engineering sciences [20-23]. Recently this method is also used to solve some of the non-linear problem in biological and physical sciences [24-27]. This method is a combination of homotopy in topology and classic perturbation techniques. Ji-Huan He used the HPM to solve the Lighthill equation [28], the Duffing equation [29] and the Blasius equation [30]. The HPM is unique in its applicability, accuracy and efficiency. The HPM uses the imbedding parameter $p$ as a small parameter, and only a few iterations are needed to search for an asymptotic solution. Using this method (see Appendix A), we can obtain the concentrations of substrate and product which are the solution to Eqs.14 and 15 (see Appendix B) as follows: 


$$
\begin{aligned}
& \bar{S}(X) \\
&=\left(\operatorname{sech} M-\frac{k_{1}}{N^{2}}+\frac{\alpha N^{2} \operatorname{sech} M}{\gamma_{S}^{2}}+\frac{\beta N^{2} \operatorname{sech} M}{\gamma_{S}^{2}}\right) \cosh M X \\
&- \frac{k_{2}(X \sinh M X)}{N^{2}}-\frac{k_{4} \cosh 3 M X}{N^{2}}-\frac{k_{7}}{N^{2}}-\frac{k_{3} \cosh 2 M X}{N^{2}} \\
& \bar{P}(X)=\left[-\frac{N^{2}(\operatorname{sech} M+1)}{M^{2}}-\left(\frac{k_{7}}{2}+\frac{k_{5}}{4}\right)\right. \\
&+\left(\frac{k_{1}}{M^{2}}+\frac{3 k_{6}}{4 M^{2}}-\frac{2 k_{2}}{M^{3}}\right)(1-\cosh M)+\left(\frac{k_{5}}{8 M^{2}}+\frac{k_{3}}{4 M^{2}}\right) \\
&\left.(1-\cosh 2 M)+\left(\frac{k_{6}}{36 M^{2}}+\frac{k_{4}}{9 M^{2}}\right)(1-\cosh 3 M)\right] X \\
&+\left[\frac{k_{5}}{4}-\frac{k_{7}}{2}\right] X^{2}+\left[-\frac{N^{2}}{M^{2}} \operatorname{sech} M+\frac{k_{1}}{M^{2}}-\frac{2 k_{2}}{M^{3}}+\frac{3 k_{6}}{4 M^{2}}\right] \\
& \cosh M X+\frac{k_{2} X \sinh M X}{M^{2}}+\left[\frac{k_{5}}{8 M^{2}}+\frac{k_{3}}{4 M^{2}}\right] \cosh 2 M X \\
&+\left[\frac{k_{4}}{9 M^{2}}+\frac{k_{6}}{36 M^{2}}\right] \cosh 3 M X+\left[\frac{2 k_{2}}{M^{3}}-\frac{k_{4}}{9 M^{2}}-\frac{k_{5}}{8 M^{2}}-\frac{k_{1}}{M^{2}}\right. \\
&\left.-\frac{3 k_{6}}{4 M^{2}}+\frac{N^{2} \operatorname{sech} M}{M^{2}}-\frac{k_{3}}{4 M^{2}}-\frac{k_{6}}{36 M^{2}}\right]
\end{aligned}
$$

where $M^{2}=\frac{\gamma_{E}^{2}}{1+\alpha+\beta}$, and

$$
\begin{aligned}
& k_{1}=\frac{N^{2} M^{3}}{2 \gamma_{E}^{2}}(\alpha+\beta) \operatorname{sech} M \tanh M \\
& +\frac{\alpha N^{2} M^{2}}{6 \gamma_{E}^{2}} \operatorname{sech}^{3} M \cosh 2 M \\
& -\frac{\beta N^{2} M^{2}}{32 \gamma_{E}^{2}} \operatorname{sech}^{3} M \cosh 3 M-\frac{3 \beta N^{2} M^{2}}{8 \gamma_{E}^{2}} \operatorname{sech}^{2} m \tanh M \\
& +\frac{\alpha N^{2} M^{2}}{2 \gamma_{E}^{2}} \operatorname{sech}^{3} M-\frac{\alpha N^{4}}{\gamma_{S}^{2}} \operatorname{sech} M-\frac{\beta N^{4}}{\gamma_{S}^{2}} \operatorname{sech} M \\
& k_{2}=-\frac{N^{2} M^{2}}{2 \gamma_{E}^{2}}(\alpha+\beta) \operatorname{sech} M+\frac{3 \beta N^{2} M^{2}}{8 \gamma_{E}^{2}} \operatorname{sech}^{2} M, \\
& k_{4}=\frac{\beta N^{2} M^{2}}{32 \gamma_{E}^{2}} \operatorname{sech}^{2} M, k_{5}=\frac{\alpha N^{4}}{\gamma_{S}^{2}} \operatorname{sech}^{2} M, \\
& k_{6}=\frac{\beta N^{4}}{\gamma_{S}^{2}} \operatorname{sech}^{2} M, k_{7}=-\frac{\alpha N^{2} M^{2}}{2 \gamma_{E}^{2}} \operatorname{sech}^{2} M, \\
& k_{3}=\frac{\alpha N^{2} M}{6 \gamma_{E}^{2}} \operatorname{sech}^{2} M,
\end{aligned}
$$

The analytical expression of dimensionless current using the Eq.17 is given by

$$
\begin{aligned}
& \psi=-\frac{N^{2}}{M^{2}}(\operatorname{sech} M+1)+\left(\frac{k_{1}}{M^{2}}+\frac{3 k_{6}}{4 M^{2}}-\frac{2 k_{2}}{M^{3}}\right) \\
& (1-\cosh M)+\left(\frac{k_{5}}{8 M^{2}}+\frac{k_{3}}{4 M^{2}}\right)(1-\cosh 2 M) \\
& +\left(\frac{k_{6}}{36 M^{2}}+\frac{k_{4}}{9 M^{2}}\right)(1-\cosh 3 M)-\left(\frac{k_{7}}{2}+\frac{k_{5}}{4}\right)
\end{aligned}
$$

\section{NUMERICAL SIMULATION}

The nonlinear differential Eqs.19 and 20 are also solved by using numerical methods. The function ode45 in Matlab software which is a function of solving two-point boundary value problems (BVPs) for ordinary differenttial equations is used to solve those equations. Its numerical solution is compared with the solution obtained by using Homotopy perturbation method and it gives a satisfactory result. The Matlab program is also given in Appendix C.

\section{DISCUSSION}

Eqs.19 and 20 are the new and simple analytical expressions of concentrations of the substrate and the product calculated using Homotopy perturbation method. The dimensionless analytical expressions of concentration substrate $\bar{S}(X)$ and product $\bar{P}(X)$ versus the dimensionless distance $X$ for various values of dimensionless reaction parameters are compared with numerical solution in Figures 1 and 2.

From these figures, it is inferred that the value of the concentration of substrate increases gradually when $X$ increases. Also the concentration of substrate increases when $\gamma_{E}$ decreases and $\alpha$ decreases. The concentration of substrate is uniform when $\gamma_{E} \leq 0.1$. From the Figure 2, it is inferred that the product have minimum value at $X=0$ and $X=1$. Also it is maximum at $X=$ 0.5. Concentration of product increases when the parameter $\gamma_{E}$ increases. Dimensionless current $\psi$ versus dimensionless parameters $\alpha, \beta, \gamma_{E}$ and $\gamma_{S}$ is plotted in Figure 3. From these figure it is inferred that the current increases when $\alpha$ and $\beta$ decreases or $\gamma_{E}$ and $\gamma_{S}$ increases.

\section{CONCLUSION}

The analytical expressions for the concentration of substrate and product in substrate inhibition process are derived using new Homotopy perturbation method. We have also presented an analytical expression for the steadystate current. The extension of procedure to various inhibition models for steady-state and non-steady state conditions seems possible. These analytical results will be useful for the optimization and the design of enzyme inhibition process. 

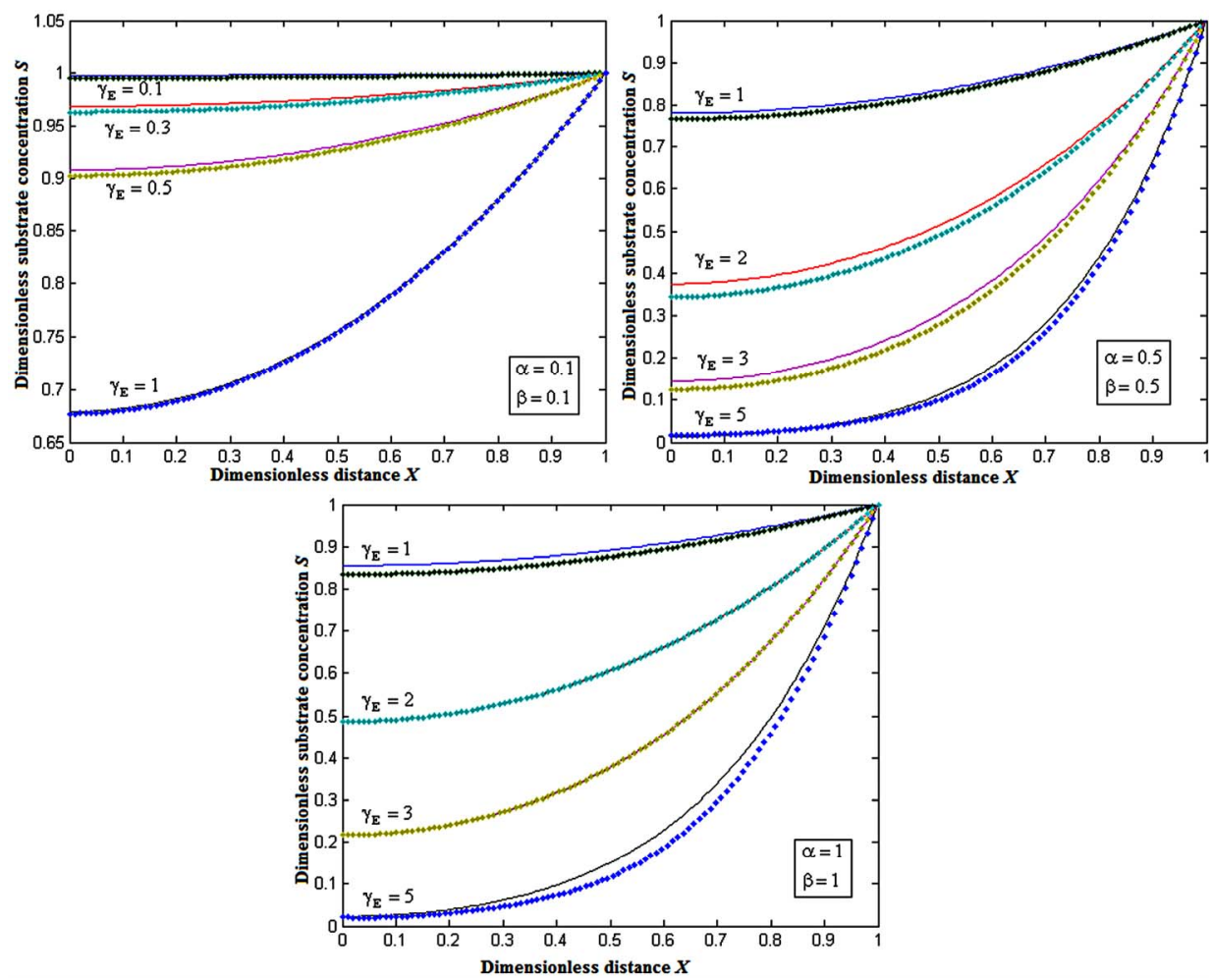

Figure 1. Plots of dimensionless concentration of substrate $S(X)$ versus dimensionless distance $X$ for various values of parameter $\gamma_{E}$ using Eq.19. The key to the plot: (••) represents Eq.19 and (-) represents numerical simulation.
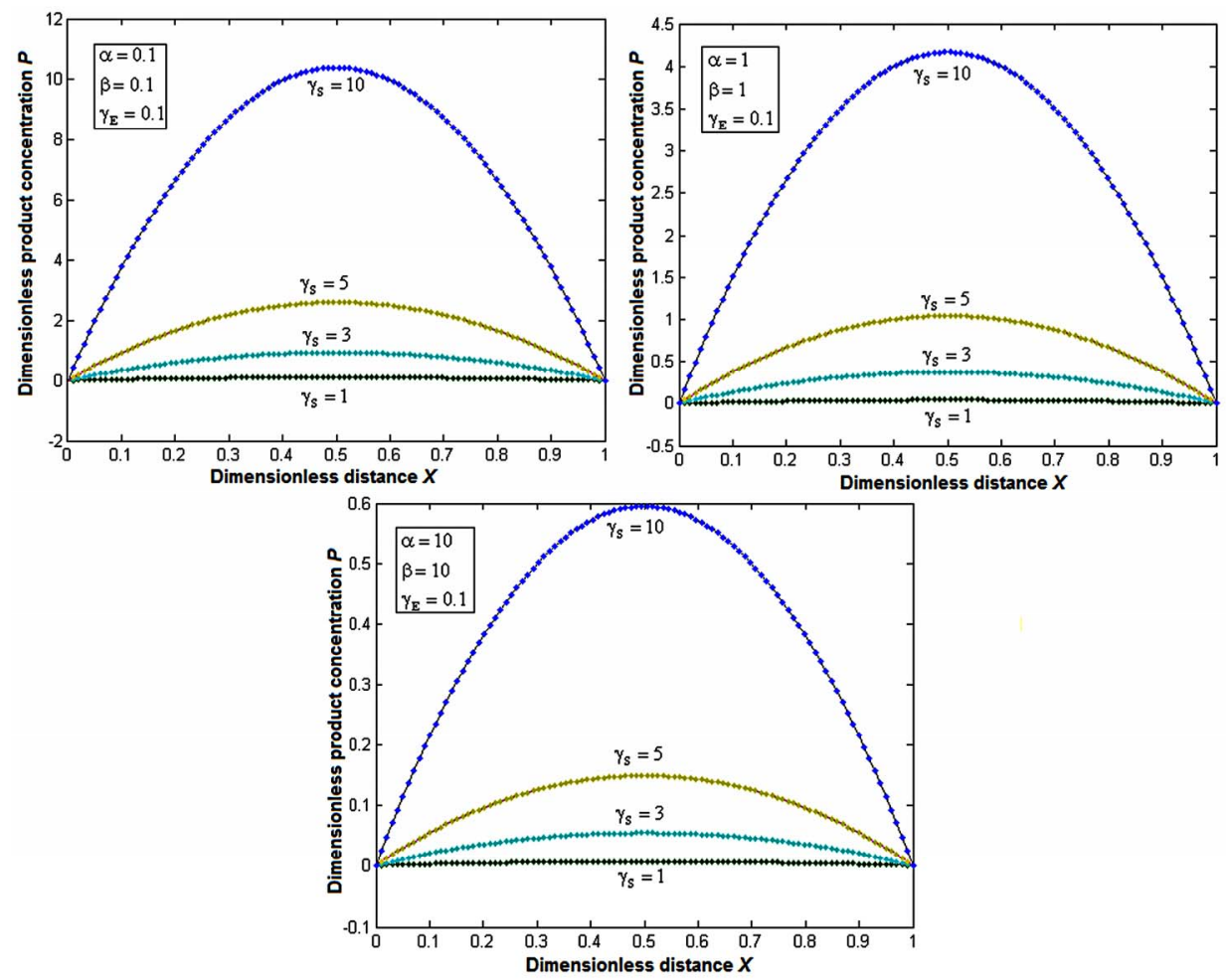

Figure 2. Plots of dimensionless concentration of product $P(X)$ versus dimensionless distance $X$ for various values of parameters $\gamma_{S}$ using Eq.20. The key to the plot: (•••) represents Eq.20 and (-) represents numerical simulation. 

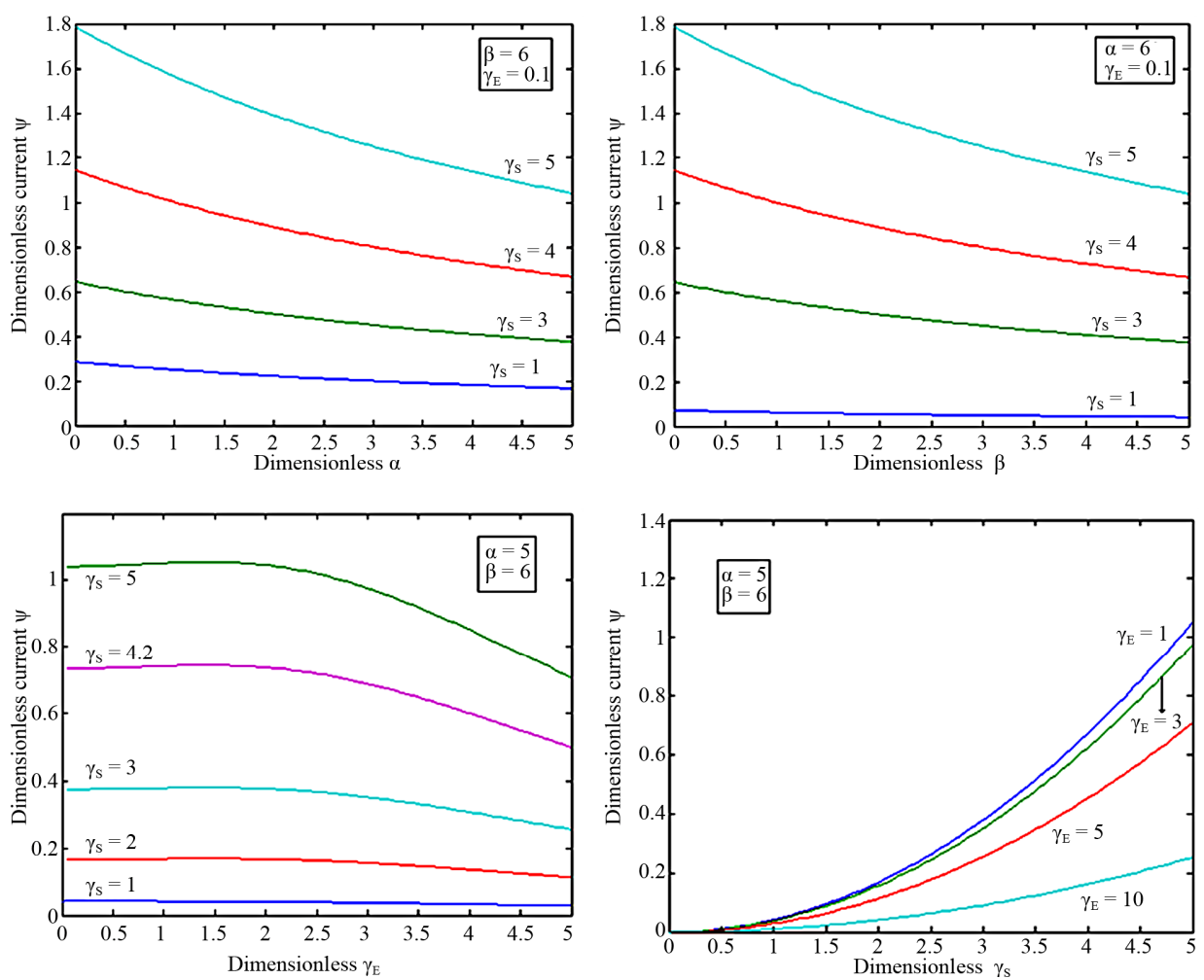

Figure 3. Plots of dimensionless current $\psi$ versus dimensionless parameters $\alpha, \beta, \gamma_{E}$ and $\gamma_{S}$ for some fixed values other parameter using the Eq.22.

\section{ACKNOWLEDGEMENTS}

This work was supported by the CSIR and DST, Government of India. The authors are also thankful to The Principal, The Madura College, Madurai and The Secretary, Madura College Board, Madurai for their encouragement.

\section{REFERENCES}

[1] Bisswanger, H. (2008) Enzyme kinetics. Principles and Methods. Wiley-VCH Verlag GmbH \& Co., Weinheim. doi:10.1002/9783527622023

[2] Dutta, R. (2008) Fundamentals of biochemical engineering. Springer, Berlin. doi:10.1007/978-3-540-77901-8

[3] House, J.E. (2007) Principles of chemical kinetics. Elsevier Inc., Amsterdam.

[4] Leskovac, V. (2004) Comprehensive enzyme kinetics. Kluwer Academic Publishers, Norwell.

[5] Missen, R.W., Mims, C.A. and Saville, B.A. (1999) Introduction to chemical reaction engineering and kinetics. John Wiley \& Sons, Inc., Hoboken.

[6] Taylor, K.B. (2002) Enzyme kinetics and mechanisms. Kluwer Academic Publishers, Norwell.

[7] Gaidamauskait, E. (2011) Computational modeling of complex reactions kinetics in biosensors. Doctoral Dissertation, Physical Sciences, Informatics (09P), Vilnius.

[8] Baronas, R., Ivanauskas, F. and Kulys, J. (2009) Mathematical modeling of bio-sensors. Springer-Verlag, Berlin.
[9] Berg, J.M., Tymoczko, J.L. and Stryer, L. (2002) Biochemistry. W. H. Freeman, New York.

[10] Rubinow, S.I. (1975) Introduction to mathematical boilogy. Wiley, New York.

[11] Murray, J.D. (1989) Mathematical biology. Springer, Berlin, 109. doi:10.1007/978-3-662-08539-4_5

[12] Segel, L.A. (1980) Mathematical models in molecular and cellular biology. Cambridge University Press, Cambridge.

[13] Roberts, D.V. (1977) Enzyme kinetics. Cambridge University Press, Cambridge.

[14] Rahamathunissa, G., Manisanar, P., Rajenran, L. and Venugopal, G. (2011) Modeling of nonlinear boundary value problems in enzyme-catalyzed reaction diffusion processes. Journal of Mathematical Chemistry, 49, 457474. doi:10.1007/s10910-010-9752-9

[15] Loghambal, S. and Rajendran, L. (2011) Mathematical modeling in amperometric oxidase enzyme-Membrane electrodes. Journal of Membrane Science, 373, 20-28.

[16] Anitha, S., Subbiah, S., Subramaniam, S. and Rajendran, L. (2011) Analytical solution of amperometric enzymatic reactions based on Homotopy perturbation method. Electrochimica Acta, 56, 3345-3352. doi:10.1016/j.electacta.2011.01.014

[17] Anitha, S., Subbiah, A. and Rajendran, L. (2011) Analytical expression of non-steady-state concentrations and current pertaining to compounds present in the enzyme membrane of biosensor, Journal of Physical Chemistry A, 115, 4299-4306. doi:10.1021/jp200520s 
[18] Uma Maheswari, M. and Rajendran, L. (2011) Analytical solution of non-linear enzyme reaction equations arising in mathematical chemistry. Journal of Mathematical Chemistry, 49, 1713-1726. doi:10.1007/s10910-011-9853-0

[19] Meena, A., Eswari, A. and Rajendran, L. (2011) Mathematical modeling of biosensors: Enzyme-substrate Interaction and bio-molecular interaction. In: Serra, P.A., Ed., New Perspectives in Biosensors Technology and Applications, InTech, Rijeka. doi:10.5772/19513

[20] Ghori, Q.K., Ahmed, M. and Siddiqui, A.M. (2007) Application of homotopy perturbation method to squeezing flow of a Newtonian fluid. International Journal of Nonlinear Sciences and Numerical Simulation, 8, 179-184.

[21] Ozis, T. and Yildirim, A. (2007) A comparative study of He's homotopy perturbation method for determining frequency-amplitude relation of a nonlinear oscillator with discontinuities. International Journal of Nonlinear Sciences and Numerical Simulation, 8, 243-248.

[22] Li, S.J. and Liu, Y.X. (2006) An improved approach to nonlinear dynamical system identification using PID neural networks. International Journal of Nonlinear Sciences and Numerical Simulation, 7, 177-182. doi:10.1515/IJNSNS.2006.7.2.177

[23] Mousa, M.M., Ragab, S.F. and Nturforsch, Z. (2008) Application of the Homotopy perturbation method to linear and nonlinear Schrödinger equations. Zeitschrift für $\mathrm{Na}$ turforschung, 63, 140-144.

[24] Margret PonRani, V. and Rajendran, L. (2010) Analytical expression of non steady-state concentration profiles at planar electrode for the CE mechanism. Natural Science, 2, 1318-1325. doi:10.4236/ns.2010.211160

[25] Varadharajan, G. and Rajendran, L. (2011) Analytical solution of coupled non-linear second order reaction differential equations in enzyme kinetics. Natural Science, 3, 459-465. doi:10.4236/ns.2011.36063

[26] Venugopal, K., Eswari, A. and Rajendran, L. (2011) Mathematical model for steady state current at ppo-modified micro-cylinder biosensors. Journal of Biomedical Science and Engineering, 4, 631-641. doi:10.4236/jbise.2011.49079

[27] Muthukumar, S. and Rajendran, L. (2012) Concentration of species in two species oscillator using Homotophy peturbation method. Global Journal of Theoretical and Applied Mathematics Sciences, 2, 99-108.

[28] He, J.H. (1999) Homotopy perturbation technique. Computer Methods in Applied Mechanics and Engineering, 178, 257-262.

[29] He, J.H. (2003) Homotopy perturbation method: A new non-linear analytical technique. Applied Mathematics and Computation, 135, 73-79. doi:org/10.1016/S0096-3003(01)00312-5

[30] He, J.H. (2003) A simple perturbation approach to Blasius equation. Applied Mathematics and Computation, 140, 217-222. doi:10.1016/S0096-3003(02)00189-3 


\section{APPENDIX A: BASIC IDEA OF HOMOTOPY PERTURBATION METHOD}

In this appendix we outline the basic idea of Homotopy perturbation method. This method has eliminated the limitations of the traditional perturbation methods. On the other hand it can take full advantage of the traditional perturbation techniques, so there has been a considerable deal of research in applying homotopy technique for solving various strongly nonlinear equations. To explain Homotopy perturbation method, let us consider the following function

$$
A(u)-f(r)=0, \quad r \in \Omega
$$

with the boundary conditions,

$$
B(u, \partial u / \partial n)=0, \quad r \in \Gamma
$$

where $A, B, f(r)$ and $\Gamma$ denote a general differential operator, a boundary operator, a known analytical function and the boundary of the domain $\Omega$, respectively. Generally speaking, the operator $A$ can be divided into a linear part $L$ and a nonlinear part $N$. Eq.A1 can therefore, be written as

$$
L(u)+N(u)-f(r)=0
$$

By Homotopy technique, we construct a Homotopy $v(r, p): \Omega \times[0,1] \rightarrow R$ which satisfies

$$
\begin{aligned}
& H(v, p)=(1-p)\left[L(v)-L\left(u_{0}\right)\right] \\
&+p[A(v)-f(r)]=0 . \\
& p \in[0,1], r \in \Omega
\end{aligned}
$$

or

$$
\begin{aligned}
H(v, p)= & L(v)-L\left(u_{0}\right)+p L\left(u_{0}\right) \\
& +p[N(v)-f(r)]=0 .
\end{aligned}
$$

where $p \in[0,1]$ is an embedding parameter, and $\mu_{0}$ is an initial approximation of Eq.A1, which satisfies the boundary conditions. When $p=0$ Eqs.A4 and A5 become a linear equation; when $p=1$ it become a nonlinear equation. So the changing process of $p$ from zero to unity is just that of changing $L(v)-L\left(u_{0}\right)=0$ to $A(v)-f(r)=0$. We can first use the embedding parameter $p$ as a small parameter. Obviously, from Eqs.A4 and $\mathbf{A 5}$, we will have

$$
\begin{aligned}
& H(v, 0)=L(v)-L\left(u_{0}\right)=0 \\
& H(v, 1)=A(v)-f(r)=0
\end{aligned}
$$

Assume that the solutions of Eqs.A4 and A5 can be written as a power series in $p$

$$
v=v_{0}+p v_{1}+p^{2} v_{2}+\cdots
$$

Setting $p=1$ results in the approximate solution of
Eq.A1:

$$
u=\lim _{p \rightarrow 1} v=v_{0}+v_{1}+v_{2}+\cdots
$$

The combination of the perturbation method and the Homotopy method is called the HPM.

\section{APPENDIX B: SOLUTION OF EQS.14 AND 15 USING HPM}

To find the solution of Eq.13, the homotopy is constructed as follows:

$$
\begin{aligned}
& (1-B)\left[\frac{\mathrm{d}^{2} \bar{S}}{\mathrm{~d} X^{2}}+\alpha \frac{\mathrm{d}^{2} \bar{S}}{\mathrm{~d} X^{2}}+\beta \frac{\mathrm{d}^{2} \bar{S}}{\mathrm{~d} X^{2}}-\gamma_{E}^{2} \bar{S}\right] \\
& +B\left[\frac{\mathrm{d}^{2} \bar{S}}{\mathrm{~d} X^{2}}+\alpha \bar{S} \frac{\mathrm{d}^{2} \bar{S}}{\mathrm{~d} X^{2}}+\beta \bar{S}^{2} \frac{\mathrm{d}^{2} \bar{S}}{\mathrm{~d} X^{2}}-\gamma_{E}^{2} \bar{S}\right]=0
\end{aligned}
$$

The approximate solution of e Eq.B1 is:

$$
\bar{S}=S_{0}+B S_{1}+B^{2} S_{2}+\cdots
$$

Substituting Eqs.B2 in B1 results:

$$
\begin{aligned}
& (1-B)\left[\frac{\mathrm{d}^{2}\left(S_{0}+B S_{1}+\cdots\right)}{\mathrm{d} X^{2}}+\alpha \frac{\mathrm{d}^{2}\left(S_{0}+B S_{1}+\cdots\right)}{\mathrm{d} X^{2}}\right. \\
& \left.+\beta \frac{\mathrm{d}^{2}\left(S_{0}+B S_{1}+\cdots\right)}{\mathrm{d} X^{2}}-\gamma_{E}^{2}\left(S_{0}+B S_{1}+\cdots\right)\right] \\
& +B\left[\frac{\mathrm{d}^{2}\left(S_{0}+B S_{1}+\cdots\right)}{\mathrm{d} X^{2}}+\alpha\left(S_{0}+B S_{1}+\cdots\right)\right. \\
& \cdot \frac{\mathrm{d}^{2}\left(S_{0}+B S_{1}+\cdots\right)}{\mathrm{d} X^{2}}+\beta\left(S_{0}+B S_{1}+\cdots\right)^{2} \frac{\mathrm{d}^{2}\left(S_{0}+B S_{1}+\cdots\right)}{\mathrm{d} X^{2}} \\
& \left.-\gamma_{E}^{2}\left(S_{0}+B S_{1}+\cdots\right)\right]=0
\end{aligned}
$$

Comparing the coefficients of the powers of $\mathrm{B}$

$$
\begin{gathered}
B^{0}: \frac{\mathrm{d}^{2} S_{0}}{\mathrm{~d} X^{2}}+\alpha \frac{\mathrm{d}^{2} S_{0}}{\mathrm{~d} X^{2}}+\beta \frac{\mathrm{d}^{2} S_{0}}{\mathrm{~d} X^{2}}-\gamma_{E}^{2} S_{0}=0 \\
B^{1}: \frac{\mathrm{d}^{2} S_{1}}{\mathrm{~d} X^{2}}+\alpha \frac{\mathrm{d}^{2} S_{1}}{\mathrm{~d} X^{2}}+\beta \frac{\mathrm{d}^{2} S_{1}}{\mathrm{~d} X^{2}}+\gamma_{E}^{2} S_{1} \\
-\alpha \frac{\mathrm{d}^{2} S_{0}}{\mathrm{~d} X^{2}}-\beta \frac{\mathrm{d}^{2} S_{0}}{\mathrm{~d} X^{2}}+\alpha S_{0} \frac{\mathrm{d}^{2} S_{0}}{\mathrm{~d} X^{2}}+\beta S_{0}^{2} \frac{\mathrm{d}^{2} S_{0}}{\mathrm{~d} X^{2}}=0
\end{gathered}
$$

The initial approximations are as follows:

$$
\begin{gathered}
X=0, \frac{\mathrm{d} S_{0}}{\mathrm{~d} x}=0, \frac{\mathrm{d} S_{1}}{\mathrm{~d} X}=0 \\
X=1, S_{0}=1, S_{1}=0
\end{gathered}
$$

Solving (B4) and (B5) with boundary conditions (B6) and (B7) gives

$$
S_{0}=\operatorname{sech} M \cosh M X
$$




$$
\begin{aligned}
S_{1}= & \left(\frac{k_{1}}{N^{2}}+\frac{\alpha N^{2}}{\gamma_{S}^{2}} \operatorname{sech} M+\frac{\beta N^{2}}{\gamma_{S}^{2}} \operatorname{sech} M\right) \cosh M X \\
& +\frac{k_{2}}{N^{2}} X \sinh M X+\frac{k_{3}}{N^{2}} \cosh 3 M X \\
& +\frac{k_{6}}{N^{2}}+\frac{k_{7}}{N^{2}} \cosh 2 M X
\end{aligned}
$$

$\bar{S}(X)=S_{0}+S_{1}$ gives the Eq.19. To find the solution of Eq.15, the homotopy is constructed as follows:

$$
\begin{aligned}
& (1-B)\left[\frac{\mathrm{d}^{2} P}{\mathrm{~d} X^{2}}+\alpha \frac{\mathrm{d}^{2} P}{\mathrm{~d} X^{2}}+\beta \frac{\mathrm{d}^{2} P}{\mathrm{~d} X^{2}}+\gamma_{S}^{2} S\right] \\
& +B\left[\frac{\mathrm{d}^{2} P}{\mathrm{~d} X^{2}}+\alpha S \frac{\mathrm{d}^{2} P}{\mathrm{~d} X^{2}}+\beta S^{2} \frac{\mathrm{d}^{2} P}{\mathrm{~d} X^{2}}+\gamma_{S}^{2} S\right]=0
\end{aligned}
$$

The approximate solution of Eq.B10 is:

$$
\bar{P}=P_{0}+B P_{1}+B^{2} P_{2}+\cdots
$$

Substituting (B11) in (B9) results:

$$
\begin{aligned}
& (1-B)\left[\frac{\mathrm{d}^{2}\left(P_{0}+B P_{1}+\cdots\right)}{\mathrm{d} X^{2}}+\alpha \frac{\mathrm{d}^{2}\left(P_{0}+B P_{1}+\cdots\right)}{\mathrm{d} X^{2}}\right. \\
& \left.+\beta \frac{\mathrm{d}^{2}\left(P_{0}+B P_{1}+\cdots\right)}{\mathrm{d} X^{2}}-\gamma_{S}^{2}\left(S_{0}+B S_{1}+\cdots\right)\right] \\
& +B\left[\frac{\mathrm{d}^{2}\left(P_{0}+B P_{1}+\cdots\right)}{\mathrm{d} X^{2}}+\alpha\left(S_{0}+B S_{1}+\cdots\right)\right. \\
& \cdot \frac{\mathrm{d}^{2}\left(P_{0}+B P_{1}+\cdots\right)}{\mathrm{d} X^{2}}+\beta\left(S_{0}+B S_{1}+\cdots\right)^{2} \frac{\mathrm{d}^{2}\left(P_{0}+B P_{1}+\cdots\right)}{\mathrm{d} X^{2}} \\
& \left.-\gamma_{E}^{2}\left(S_{0}+B S_{1}+\cdots\right)\right]=0
\end{aligned}
$$

Comparing the coefficients of the powers of $B$

$$
\begin{gathered}
B^{0}: \frac{\mathrm{d}^{2} P_{0}}{\mathrm{~d} X^{2}}+\alpha \frac{\mathrm{d}^{2} P_{0}}{\mathrm{~d} X^{2}}+\beta \frac{\mathrm{d}^{2} P_{0}}{\mathrm{~d} X^{2}}-\gamma_{S}^{2} S_{0}=0 \\
B^{1}: \frac{\mathrm{d}^{2} P_{1}}{\mathrm{~d} X^{2}}+\alpha \frac{\mathrm{d}^{2} P_{1}}{\mathrm{~d} X^{2}}+\beta \frac{\mathrm{d}^{2} P_{1}}{\mathrm{~d} X^{2}}+\gamma_{S}^{2} S_{1} \\
-\alpha \frac{\mathrm{d}^{2} P_{0}}{\mathrm{~d} X^{2}}-\beta \frac{\mathrm{d}^{2} P_{0}}{\mathrm{~d} X^{2}}+\alpha S_{0} \frac{\mathrm{d}^{2} P_{0}}{\mathrm{~d} X^{2}}+\beta S_{0}^{2} \frac{\mathrm{d}^{2} P_{0}}{\mathrm{~d} X^{2}}=0
\end{gathered}
$$

The initial approximations are as follows

$$
\begin{array}{r}
\text { At } X=0, P_{0}=0, P_{1}=0 \\
\text { At } X=1, P_{0}=0, P_{1}=1
\end{array}
$$

Solving (B13) and (B14) with boundary conditions (B15) and (B16) gives

$$
P_{0}=-\frac{N^{2}}{M^{2}}(\operatorname{sech} M \cosh M X+(\operatorname{sech} M+1) X-\operatorname{sech} M)
$$

$$
\begin{aligned}
& P_{1}=\left[\left(\frac{k_{1}}{M^{2}}+\frac{3 k_{6}}{4 M^{2}}-\frac{2 k_{2}}{M^{3}}\right)(1-\cosh M)\right. \\
& +\left(\frac{k_{5}}{8 M^{2}}+\frac{k_{3}}{4 M^{2}}\right)(1-\cosh 2 M) \\
& \left.+\left(\frac{k_{6}}{36 M^{2}}+\frac{k_{4}}{9 M^{2}}\right)(1-\cosh 3 M)-\left(\frac{k_{7}}{2}+\frac{k_{5}}{4}\right)\right] X \\
& +\left[\frac{k_{5}}{4}+\frac{k_{7}}{2}\right] X^{2}+\left[\frac{k_{1}}{M^{2}}-\frac{2 k_{2}}{M^{3}}+\frac{3 k_{6}}{4 M^{2}}\right] \cosh M X \\
& +\frac{k_{2} X \sinh M X}{M^{2}}+\left[\frac{k_{5}}{8 M^{2}}+\frac{k_{3}}{4 M^{2}}\right] \cosh 2 M X \\
& +\left[\frac{k_{4}}{9 M^{2}}+\frac{k_{6}}{36 M^{2}}\right] \cosh 3 M X+\left[\frac{2 k_{2}}{M^{3}}-\frac{k_{4}}{9 M^{2}}-\frac{k_{5}}{8 M^{2}}\right. \\
& \left.-\frac{k_{1}}{M^{2}}-\frac{3 k_{6}}{4 M^{2}}+\frac{N^{2} \sec h M}{M^{2}}-\frac{k_{3}}{4 M^{2}}-\frac{k_{6}}{36 M^{2}}\right]
\end{aligned}
$$

$\bar{P}(X)=P_{0}+P_{1}$ gives the Eq.20.

\section{APPENDIX C: MATLAB PROGRAM TO FIND THE NUMERICAL SOLUTION OF EQS.14 AND 15}

function pdex3

$\mathrm{m}=0$;

$\mathrm{x}=$ linspace $(0,1)$;

$\mathrm{t}=$ linspace $(0,100000000)$;

sol=pdepe(m,@pdex4pde,@pdex4ic,@pdex4bc,x,t);

u1 = sol(:::,1);

$\mathrm{u} 2=\operatorname{sol}(:,:, 2)$;

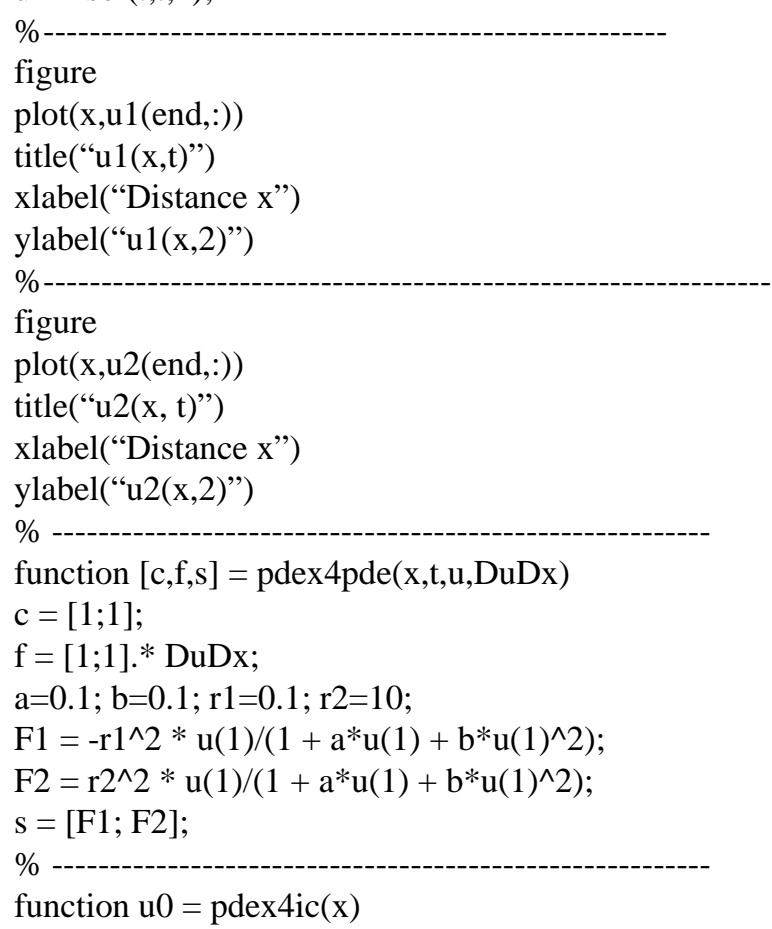


$\mathrm{u} 0=[1 ; 1]$

$\%$ -

function $[\mathrm{pl}, \mathrm{ql}, \mathrm{pr}, \mathrm{qr}]=\operatorname{pdex} 4 \mathrm{bc}(\mathrm{xl}, \mathrm{ul}, \mathrm{xr}, \mathrm{ur}, \mathrm{t})$ $\mathrm{pl}=[0 ; \mathrm{ul}(2)-0]$;

$$
\begin{aligned}
\mathrm{ql} & =[1 ; 0] ; \\
\mathrm{pr} & =[\operatorname{ur}(1)-1 ; \operatorname{ur}(2)-0] ; \\
\mathrm{qr} & =[0 ; 0]
\end{aligned}
$$

